Research

Article

\title{
A Meta-Modeling Approach for Autonomous Driving Scenario Based on STTD
}

\author{
Menghan Zhang (张梦寒) ${ }^{1,2,3}$, Dehui Du (杜德慧) $)^{1,2,3}$, Mingzhuo Zhang \\ $(\text { 张铭茁 })^{1,2,3}$, Lei Zhang (张雷 $)^{4}$, Yao Wang $(\text { 王耀 })^{1,2,3}$, Wentao Zhou (周文韬 $)^{1,2,3}$ \\ 1 (Software Engineering Institute, East China Normal University, Shanghai 200062, China) \\ 2 (Shanghai Key Laboratory of Trustworthy Computing (East China Normal University), Shanghai 200062, \\ China) \\ 3 (International Joint Laboratory of Trustworthy Software of Ministry of Education (East China Normal \\ University), Shanghai 200062, China) \\ 4 (Shanghai Research Institute for Intelligent Autonomous Systems, Tongji University, Shanghai 201210, \\ China) \\ Corresponding author: Dehui Du,dhdu@sei.ecnu.edu.cn
}

\begin{abstract}
In the current autonomous driving scenario modeling and simulation field, autonomous driving modeling driven by Spatio-Temporal Trajectory Data (STTD) is a key problem, which is significant to improve the safety of the system. In recent years, great progress has been achieved in the modeling and application of STTD, and the application of this data in specific fields has attracted wide attention. However, because STTD has diversity and complexity as well as massive, heterogeneous, dynamic characteristics, the research in the safety-critical field modeling still faces challenges, including unified metadata of spatio-temporal trajectories, meta-modeling methods based on STTD, data processing based on the data analysis of spatiotemporal trajectories, and data quality evaluation. In view of the modeling requirements in the field of autonomous driving, a meta-modeling approach is proposed to construct spatiotemporal trajectory metadata based on Meta Object Facility (MOF) meta-modeling system. According to the characteristics of spatio-temporal trajectory data and autonomous driving domain knowledge, a meta-model of spatio-temporal trajectory data is constructed. Then, we study the modeling approach of autonomous driving safety-critical scenarios based on the spatio-temporal trajectory data meta-modeling technology system, use the modeling language ADSML for automatic instantiation of safety-critical scenarios, and construct a library of safetycritical scenarios, aiming to provide a feasible approach for the modeling of such safety-critical scenarios. Combined with the scenarios of lane changing and overtaking, the effectiveness of the meta-modeling method for autonomous driving safety scenarios driven by spatio-temporal trajectory data is demonstrated, which lays a solid foundation for the construction, simulation, and analysis of the model.
\end{abstract}

Keywords Spatio-Temporal Trajectory Data (STTD); meta-model of spatio-temporal trajectory data; domain modeling; MOF meta-modeling; autonomous driving scenario modeling

This is the English version of the Chinese article “时空轨迹数据驱动的自动驾驶场景元建模方法. 软件学报, 2021, 32(4): 973-987. doi: 10.13328/j.cnki.jos.006226”.

Funding items: National Natural Science Foundation of China (61972153); National Key R\&D Program of China (2018YFE 0101000); Key Projects of the Ministry of Science and Technology (2020AAA0107800)

Received 2020-09-13; Revised 2020-10-26; Accepted 2020-12-19; IJSI published online 2021-09-25 
Citation Zhang MH, Du DH, Zhang MZ, Zhang L, Wang Y, Zhou WT. A meta-modeling approach for autonomous driving scenario based on STTD, International Journal of Software and Informatics, 2021, 11(3): 315-333. http://www.ijsi.org/1673-7288/262.htm

\section{Introduction}

The rapid development of big data, artificial intelligence, autonomous driving, smart city, intelligent transportation, and other fields in recent years has promoted the widespread application of the next-generation information technology. Therefore, using the generated massive data for modeling of safety-critical scenarios has become an urgent problem to be solved. Integrating spatio-temporal data generated by global observation with public media data (e.g., urban cameras, social media, and personal activities) for effective spatio-temporal fusion, high-confidence modeling, big data processing, and spatio-temporal data application in specific fields has become a research hotspot in the fields of spatial information technology and big data application. The advancing integrated technologies such as Global Navigation Satellite System (GNSS), China's Remote Sensing (RS) satellites, and Geographic Information System (GIS), and Smart City enrich the spatio-temporal data which show high spatial resolution, high temporal resolution, high spectral resolution, high-precision spatio-temporal identification, and multi-dimensional attributes. Spatio-temporal trajectory data is a special kind of spatio-temporal data, which covers the information of geometry, spectrum, behavior, and semantic association, being massive, heterogeneous, and dynamic and reflecting the diversity and complexity of the real world. Therefore, multi-level analysis and data-driven application development such as data modeling, description, evaluation and verification have become an important way to perceive, recognize, and control the data for the real world ${ }^{[1-3]}$.

The rapid development of the autonomous driving technology has attracted wide spread attention from academia and industry, while it has also brought a series of safety issues. For example, a Tesla Model X went out of control on September 5, 2020 and caused 2 deaths and 6 injuries in a tragic traffic accident ${ }^{[4]}$. This series of traffic accidents caused by autonomous driving vehicles has alarmed people and indicates there is still a long way to go before autonomous vehicles officially enter the market, and a large number of experiments are needed to ensure the safety of autonomous driving. According to the research report of the RAND Corporation ${ }^{[5]}$, hundreds of billions of miles of public road tests need to be carried out to demonstrate the reliability of autonomous vehicles in avoiding deaths or injuries. However, the increasing level and scale of autonomous driving makes it unpractical to conduct the actual vehicle tests. The simulation test uses some algorithms, simulation software, and simulation parameters to mimic the virtual scenarios, but the obtained data cannot completely represent the real driving data of autonomous vehicles ${ }^{[6]}$. Therefore, to accelerate the fast and safe driving of autonomous vehicles on the road, researchers should not only rely on simulation tests but also improve the accuracy of the autonomous vehicle modeling and the effectiveness of data analysis. The mining and utilization of high-quality spatio-temporal trajectory data is the key to realizing the real scenario modeling of autonomous driving ${ }^{[7,8]}$. However, the available modeling methods and technologies for real data still have shortcomings. The available studies of autonomous driving modeling mainly focus on the design of modeling scenarios and the discussion of simulation results. However, there is not a set of modeling methods for the systematic model-driven and data-driven collaborative development. This is not convenient for the management of real data or the generation of high-quality data besides, there are two other problems: lack of the scenario description language with a good readability, and the difficulty of formal verification. Although GeoScenario ${ }^{[9]}$ and Scenic ${ }^{[10]}$ are more concise than other 
scenario modeling languages and have improved readability, their description methods are not friendly enough to domain experts that cannot use formal methods to verify and analyze the scenarios. In short, there is an urgent need for a set of spatio-temporal trajectory data-driven autonomous driving scenario modeling methods to abstractly model autonomous driving in the real world to construct a specific safety scenario library, so as to lay a foundation for modeling, simulation, and verification of autonomous driving.

In response to the above problems, this paper proposes a method for modeling autonomous driving scenarios driven by spatio-temporal trajectory data to guide the modeling, simulation, and verification of safety-critical scenarios and the construction of a safety scenario library. With this method, spatio-temporal trajectory data can be better applied to the modeling of autonomous driving, which can provide a feasible solution for the data-driven and model-driven collaborative development in the field of autonomous driving. An autonomous vehicle driving in a real environment will generate the trajectory route. The specific spatio-temporal trajectory data generated by the static and dynamic scenarios around the trajectory route can be obtained to support the autonomous driving modeling. First, Open Street Map (OSM) ${ }^{[11]}$ is used to extract the road network structure and build a static scenario model. The map matching algorithm is employed to match the GPS location points of the autonomous vehicle to the road network model and then technologies like on-board sensors and Internet of Vehicles are used to collect the surrounding dynamic information (including other vehicles and pedestrians). The relevant speed limits, traffic control conditions, and traffic congestion conditions of the road network can be obtained from the relevant departments. After data cleaning, fusion, and unified expression, the real-world scenario trajectory data set is obtained. In the end, an autonomous driving scenario modeling language is used to automatically instantiate the method. The structured trajectory data set is input into the autonomous driving model for the automatic instantiation of the modeling of autonomous driving. At the same time, the operation of the autonomous driving vehicle in multiple scenarios is instantiated for the construction of the corresponding autonomous driving safety-critical scenario library and providing the data support for further work.

In summary, the main contribution of this paper includes the following three aspects.

(1) An autonomous driving scenario modeling method driven by spatio-temporal trajectory data is proposed. This method supports the model-driven and data-driven collaborative development, and provides a novel research idea for scenario modeling in the field of autonomous driving.

(2) A meta-modeling system of spatio-temporal trajectory data is proposed based on Meta Object Facility $(\mathrm{MOF})^{[12,13]}$, which constructs a spatio-temporal trajectory data meta-model with the domain knowledge in autonomous driving and guides the domain modeling driven by spatio-temporal trajectory data.

(3) With the results of our previous research, namely the Autonomous Driving Scenario Modeling Language (ADSML) ${ }^{[14]}$, automatic instantiation of autonomous driving safety scenarios is realized to build a scenario model library for autonomous driving.

Section 2 of this paper introduces the spatio-temporal trajectory data-driven meta-modeling method of autonomous driving safety scenarios. Section 3 introduces the meta-modeling method of spatio-temporal trajectory data, including the structure of spatio-temporal trajectory metadata, the MOF-based meta-modeling system of spatio-temporal trajectory data, the meta-model of spatio-temporal trajectory data, and the preprocessing and quality evaluation of data. With the scenario modeling language ADSML, Section 4 automatically instantiates the spatio-temporal trajectory data-driven autonomous driving scenario model based on the meta-model of spatiotemporal trajectory data. The practical application shows that the method is feasible. Section 5 
compares and analyzes the related work. Finally, the full paper is summarized, and the future research directions worthy of attention are discussed.

\section{STTD-Driven Autonomous Driving Scenario Meta-Modeling Approach}

Considering the problems faced by safety scenario modeling, this paper proposes a method for autonomous driving modeling driven by spatio-temporal trajectory data. The core idea of this method is to abstract the meta-model of Spatio-Temporal Trajectory Data (STTD) of autonomous driving from the complex spatio-temporal trajectory data with consideration to the requirements and international standards ${ }^{[15-18]}$ of the data-driven engineering and autonomous driving safety scenario field. We then construct a knowledge base of spatio-temporal trajectory data based on its data set, combine the spatio-temporal trajectory data with the domain model, and design and develop highly reliable autonomous driving software by using a collaborative development method driven by data and model. Specifically, according to the MOF meta-modeling theory, a meta-model is constructed for multi-dimensional and heterogeneous spatio-temporal trajectory data of GIS, GNSS, and RS. A unified representation form of the data is constructed to support the fusion, conversion, and sharing of spatio-temporal trajectory data. Furthermore, under the guidance of spatio-temporal trajectory data meta-modeling, a scenario model oriented to autonomous driving is constructed, and the modeling framework is finally built.

As shown in Figure 1, the modeling framework mainly includes seven modules: collection of STTD, preprocessing and quality evaluation of STD, MOF-based meta-modeling system of spatio-temporal trajectory data, meta-model construction of spatio-temporal trajectory data, modeling language design of autonomous driving safety-critical scenarios, modeling and library construction of autonomous driving safety-critical scenarios, and the specification and verification of autonomous driving safety-critical scenarios. The discussion in this paper focuses on the third and fourth modules; the seven modules are briefly described below.

(1) Collection of spatio-temporal trajectory data: Measurable and non-measurable data with the time and space relationships is collected from GNSS, space-ground integrated RS, 3D Geographic Information System (3DGIS), OSM, Internet of Things (IoT), Vehicle to X (V2X), on-board sensors, and Autonomous Driving System (ADS) to form spatio-temporal trajectory data, including temporal data, spatial data, spectral data, humanistic data, social data, data quality, autonomous driving domain knowledge, and other data. Based on the knowledge and requirements of autonomous driving, the structure of the spatio-temporal trajectory metadata is abstracted to guide the processing and application of spatio-temporal trajectory data. This module will be introduced in detail in Sections 3.1 and 3.4.

(2) Preprocessing and quality evaluation of spatio-temporal trajectory data: After cleaning, fusion, and unified expression of spatio-temporal trajectory data, a quality evaluation model for spatio-temporal trajectory data is proposed to provide the quality evaluation standards for the processed spatio-temporal trajectory data, thereby ensuring the data quality. This module will be introduced in detail in Section 3.4.

(3) MOF-based meta-modeling system for spatio-temporal trajectory data: Based on the MOF modeling framework, the meta-modeling system and the hierarchical division of metamodeling for spatio-temporal trajectory data are proposed, which lays a foundation for the modeling, exchange, sharing, and interoperation of metadata. In addition, the meta-modeling system based on spatio-temporal trajectory data can combine different domain knowledge to design and develop the corresponding languages to realize the domain modeling of data-driven and model-driven collaborative development. This module will be discussed in Section 3.2. 


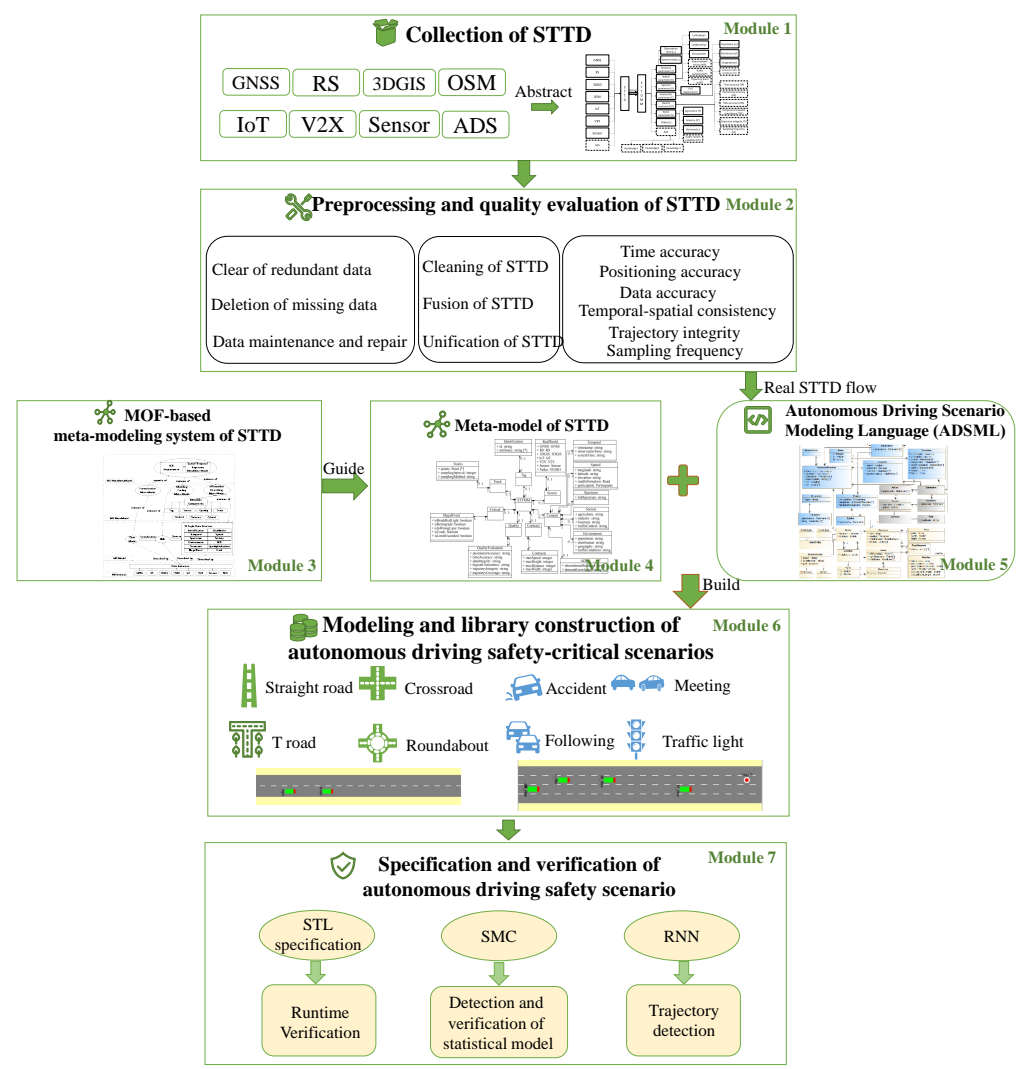

Figure 1 Meta-modeling framework of autonomous driving safety-critical scenarios based on spatiotemporal trajectory model

(4) Construction of the spatio-temporal trajectory data meta-model: Combining the domain knowledge model of autonomous driving with the spatio-temporal trajectory data meta-modeling system, one can construct a spatio-temporal trajectory data meta-model to guide the modeling and analysis of spatio-temporal trajectory data of autonomous driving, which is used for data input of scenario models. This module will be discussed in detail in Section 3.3.

(5) Language design for autonomous driving safety-critical scenario modeling: We had developed the scenario modeling language ADSML in our previous work. Section 4.1 will briefly describe the syntax, semantics, and graphical modeling of ADSML to help modelers to build the safety-critical scenario model of autonomous driving.

(6) Modeling and library construction of autonomous driving safety-critical scenarios: High-quality spatio-temporal trajectory data and the autonomous driving safety-critical scenario modeling language are combined to instantiate the scenario models and then to construct a safety-critical scenario library, which lays a foundation for the specification and verification of autonomous driving safety-critical scenarios. This module will be discussed in Section 4.2.

(7) Specification and verification of autonomous driving safety-critical scenario: This module uses the Signal Temporal Logic (STL) ${ }^{[19]}$ to specify the requirements of scenarios, and supports the verification and analysis of their safety based on the statistical model checking technology and the scenario library. This will help to carry out the preliminary verification of safe autonomous driving and clarify the role of the scenario library. This will be the focus of 
the next-phase research.

The safety-critical scenario meta-modeling method driven by spatio-temporal trajectory data covers the entire process of modeling, simulation, and verification in the field of autonomous driving, and provides technical support for safety-critical scenario simulation before the industrialization of autonomous driving. This paper will focus on the adaptation of this method to safety-critical scenario meta-modeling driven by spatio-temporal trajectory data, which is one of the main contributions of this paper.

\section{Meta-Modeling of STTD for Autonomous Driving}

According to the autonomous driving scenario meta-modeling driven by spatio-temporal trajectory data, this section will focus on how to construct the metadata structure of the spatiotemporal trajectory based on the domain characteristics of autonomous driving, the MOF-based meta-modeling system of spatio-temporal trajectory data, and the meta-model of spatio-temporal trajectory data. Finally, a high-quality evaluation method of spatio-temporal trajectory data is given.

\subsection{Metadata structure of spatio-temporal trajectory}

The spatio-temporal trajectory data has diversity and complexity as well as massive, heterogeneous, and dynamic characteristics. Therefore, efforts should be made to deeply mine the valuable information in the spatio-temporal trajectory data and efficiently process the data, so as to model the real scenario of autonomous driving. The spatio-temporal trajectory data of autonomous driving proposed in this paper not only includes the trajectory data of the autonomous vehicle, but also includes the static information (e.g., spatial information, spectral information, humanistic information, and social information) around the vehicle and the dynamic environmental information (e.g., the information of traffic participants). The metadata structure of the spatio-temporal trajectory data is shown in Figure 2.

The metadata structure of the spatio-temporal trajectory is the basis for the construction and application of spatio-temporal trajectory data ${ }^{[2,3]}$. With the spatial position and observation time information obtained by GNSS, the metadata can be expressed as the GNSS info = $f\left(\varphi, \mu, H, t_{p}, \pi\right)$, where $(\varphi, \mu, H)$ represents the latitude, longitude, and elevation, respectively; $\left(t_{p}, \pi\right)$ represents the observation time and other parameters of the GNSS receiver. Using RS equipment (space-based, air-based, and ground-based) to achieve earth observation, we can express the obtained ground object spectrum information as the RS info $=f\left(x, y, z, \lambda, t_{R}\right)$, where $(x, y)$ are the parameters of the spatial position; $(z)$ is the observation value corresponding to $(x, y)$ and related to the spatial resolution; $(\lambda)$ is the electromagnetic wave band used (related to the spectral resolution); $\left(t_{R}\right)$ is the repeated observation period for the same target object on the ground. For GIS, it can be expressed as the GIS info $=\left\{i, j, T(A), t_{G}\right\}$, where $(i, j)$ are the spatial position coordinates adopted by the system; $T(A)$ is the spatial characteristics and attributes corresponding to the system coordinates $(i, j) ;\left(t_{G}\right)$ is the time feature of the system information. The road network information is obtained using the OSM and expressed as the OSM info $=(R)$, where $R$ represents the obtained road network data. The humanistic and social data from IoT and social media can be expressed as the More info $=\{C(\alpha, \beta, \tau, H T), S(\gamma, \psi, \omega, T C), t, \pi\}$, where $C(\alpha, \beta, \tau, H T)$ is the parameter and information collection of humanistic attributes; $S(\gamma, \psi, \omega, T C)$ is the parameter and information collection of social attributes; $t$ is the time of information collection; $\pi$ is other multi-source data. The information obtained by on-board sensors and V2X is represented as $S V=\{P, G\}$, where $P$ and $G$ respectively represent the collected traffic participants and the set of trajectory points. ADS is a knowledge model in the field of autonomous driving. Quality evaluation 
model $D$ represents the Time Accuracy (TA), Positioning Accuracy (PA), Data Accuracy $(D A)$, Temporal and Spatial Consistency (TSC), Trajectory Integrity (TI), and Sampling Frequency $(S F)$.

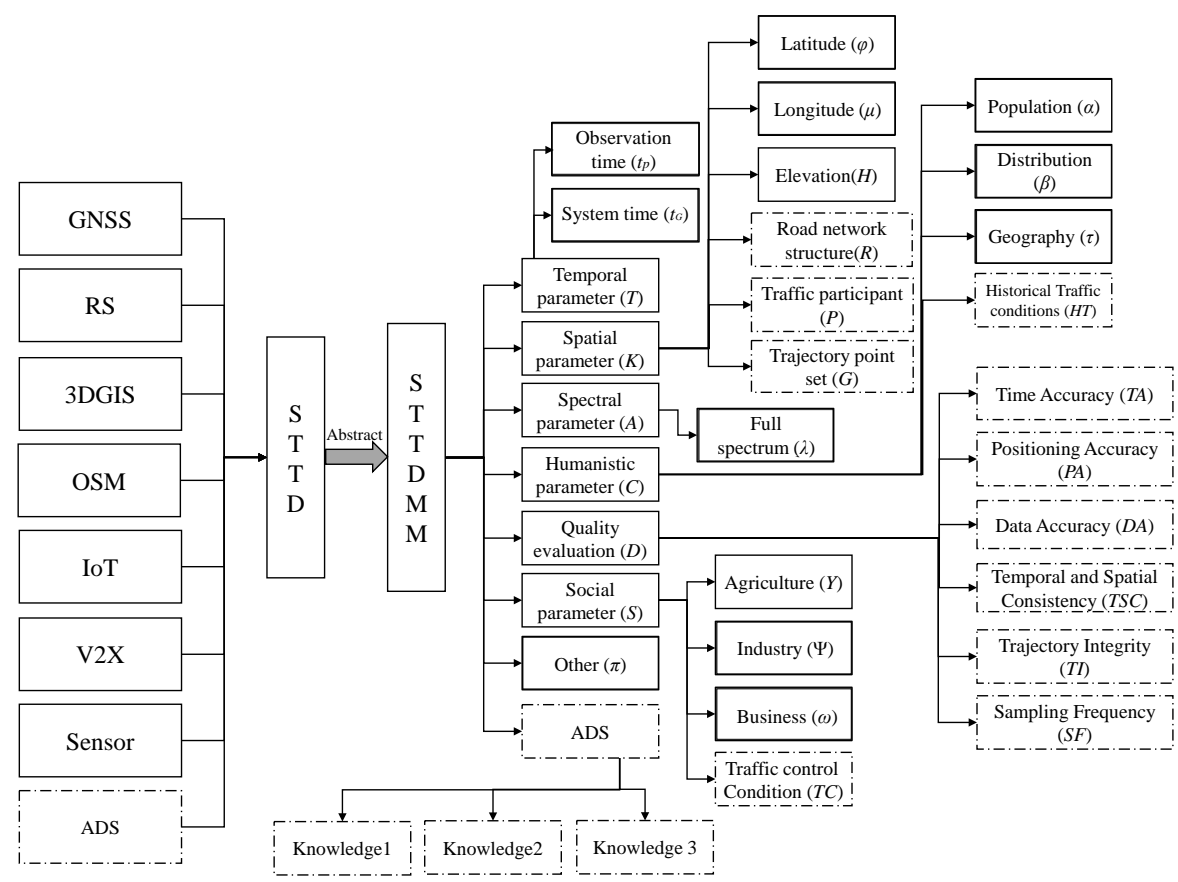

Figure 2 Metadata structure of spatio-temporal trajectory

Therefore, the spatial relationship $K(\varphi, \mu, H, R, P, G)$, temporal relationship $T\left(t_{p}, t_{G}\right)$, spectral feature $A(\lambda)$, humanistic attribute $C(\alpha, \beta, \tau, H T)$, social attribute $S(Y, \psi, \omega, T C)$, quality evaluation model $D(T A, P A, D A, T S C, T I, S F)$, autonomous driving domain knowledge ADS, and other data $\pi$ of the information carrier can be uniformly described. The unified spatiotemporal metadata expression: Spatio-Temporal Metadata $=f\left\{K(\varphi, \mu, H, R, P, G), T\left(t_{p}\right.\right.$, $\left.\left.t_{G}\right),(\lambda), C(\alpha, \beta, \tau, H T), S(Y, \psi, \omega, T C), D(T A, P A, D A, T S C, T I, S F), A D S, \pi\right\}$ is constructed to perform the dynamic management of multi-temporal, multi-scale, multi-type, multi-source spatio-temporal information. The precision of temporal resolution, spatial resolution, spectral resolution, and geographic indication is comprehensively analyzed. The metadata structure of spatio-temporal trajectory is a structured description of trajectory data, which lays a foundation for the meta-modeling of autonomous driving scenarios and is the key to ensuring data quality.

\subsection{MOF-based meta-modeling system for STTD}

This paper establishes a meta-modeling system for spatio-temporal trajectory data based on MOF. Specifically, the MOF-based meta-modeling system includes four layers: meta-metamodel layer M3, meta-model layer M2, model layer M1, and instance layer M0, and the abstraction degree gradually increases from M0 to M3. The layer division of the meta-modeling lays a foundation for the modeling, exchange, sharing, and interoperation of the metadata. In addition, based on this system, different domain knowledge can be combined for the design and development of domain modeling languages and the domain modeling by data-driven and model-driven collaborative development. 
Figure 3 shows the MOF-based meta-modeling system for spatio-temporal trajectory data proposed in this paper, which is composed of M3 to M0 layers, as described as follows.

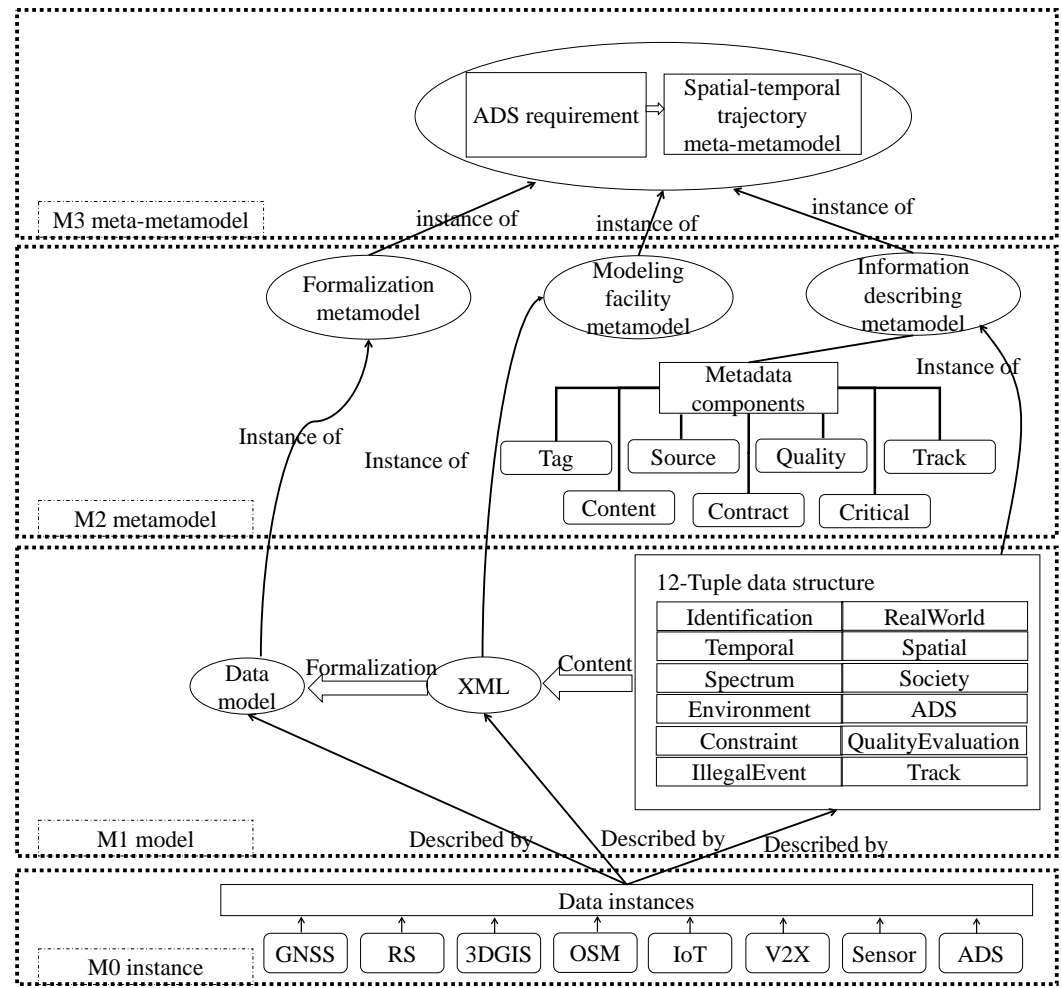

Figure 3 MOF-based meta-modeling system of spatio-temporal trajectory data

(1) Meta-metamodel layer M3: M3 is a highly abstracted meta-metamodel of spatiotemporal trajectory data, and defines the related concepts, requirements, and abstract semantics of autonomous driving scenario modeling driven by spatio-temporal trajectory data.

(2) Meta-model layer M2: M2 is an instance of the meta-metamodel. According to the development standards of meta-model in software engineering specified by OMG, the metametamodel is divided into three modules: data meta-model module, tool meta-model module, and data storage meta-model module. In the data meta-model module, metadata are abstracted according to the requirements, concepts, and characteristics of autonomous driving ${ }^{[14]}$, including seven categories: tag, source, content, contract, quality, critical, and track. The information basically covers the data necessary in the field of automatic driving safety scenario modeling. The tool meta-model module designs the interface implementation standards for obtaining the spatio-temporal trajectory data of the safety-critical scenario, which can process the data more conveniently and provide continuous data flow input for the scenario modeling. The data storage meta-model module establishes the data storage specifications, which can make the data presented in a readable form.

(3) Model layer M1: M1 is not only the instantiation of the meta-model but also the formatted expression and storage of instance layer data. It expresses the M2 layer more intuitively as data structure, XML document model, and data model. For autonomous driving safety scenario modeling, M1 concretizes the meta-data in M2 layer and provides a data structure with twelve 
elements: Identification, RealWorld, Temporal, Spatial, Spectrum, Society, Environment, ADS, Contracts, IllegalEvent, Tracks, and Quality Evaluation. Based on the XML encoding format, an XML document model is designed for the above data structure to store the high-quality spatiotemporal trajectory data. The above XML document model is represented as a spatio-temporal trajectory data model.

(4) Instance layer M0: M0 provides the data sources for the model according to the data structure required by the M1 layer and points out that the 12-dimensional spatio-temporal trajectory data mainly comes from GNSS, RS, 3DGIS, OSM, IoT, V2X, Sensor, and ADS. This layer provides the methods for the collection and processing of spatio-temporal trajectory data.

The above MOF-based meta-modeling system of spatio-temporal trajectory data provides data support and technical reference for autonomous driving modeling from data collection and preprocessing, data model, data storage, and data meta-model.

\subsection{Meta-model of spatio-temporal trajectory data}

In the model-driven software development, meta-modeling refers to the abstract representation of "concepts" in a specific field. The meta-model abstracts the common and essential modeling elements based on the model, namely the model of the model. The metadata structure of the spatio-temporal trajectory introduced in Section 3.1 can guide the modeler to capture the required data from the massive data collected in the autonomous driving field. By encapsulating the captured data into a spatio-temporal trajectory data meta-model, the modelers can build an autonomous driving model from a model-driven perspective, thereby providing structured data for autonomous driving modeling tools. Therefore, the MOF-based meta-modeling system of spatio-temporal trajectory data described in Section 3.2 is used to abstract the meta-model, as shown in Figure 4.

Figure 4 illustrates the spatio-temporal trajectory data meta-model based on the autonomous driving safety-critical scenario modeling. In order to facilitate modeling, simulation, and verification, we took the seven categories (tag, source, content, contract, quality, critical, and track) of data meta-models as the core part of the spatio-temporal trajectory data meta-model under the guidance of the content introduced in Section 3.2. On this basis, the 12-dimensional spatio-temporal trajectory data is introduced to jointly constitute the spatio-temporal trajectory data meta-model, which includes Identification, RealWorld, Temporal, Spatial, Spectrum, Society, Environment, ADS, Contracts, IllegalEvent, Tracks, and QualityEvaluation.

Tag is refined into identification, which mainly describes the characteristic information in the autonomous driving safety-critical scenario. Source is refined into real data, which records the source and the method for obtaining spatio-temporal trajectory data from the real world, such as GNSS. Content is refined into temporal parameters, spatial parameters, spectral parameters, social parameters, humanistic parameters, and autonomous driving domain knowledge. Among them, temporal parameters include timestamp, observation time, and system time. Spatial parameters include longitude, latitude, elevation, road information, and surrounding pedestrians. Spectral parameters include the full spectrum. Society parameters include agriculture, industry, business, and traffic control. Environment parameters include population, distribution, geography, and traffic condition. ADS includes international standard ISO 26262 and domain knowledge. Constraints exist in the autonomous driving safety-critical scenario, including the maximum speed, height, width of the vehicle, and the distance between vehicles. Data quality is refined into the quality evaluation of spatio-temporal trajectory data, which describes the 6-dimensional quality evaluation indicators (time accuracy, positional accuracy, data accuracy, temporal and spatial consistency, trajectory integrity, and sampling frequency) of spatio-temporal trajectory data. Safety-critical is refined into IllegalEvent, which 
indicates whether the autonomous driving scenario is in a safe state or not and records several typical illegal scenarios. Tracks are refined into the trajectory features, which mainly describe the trajectory of autonomous vehicles, including the points, intervals, and methods of sampling.

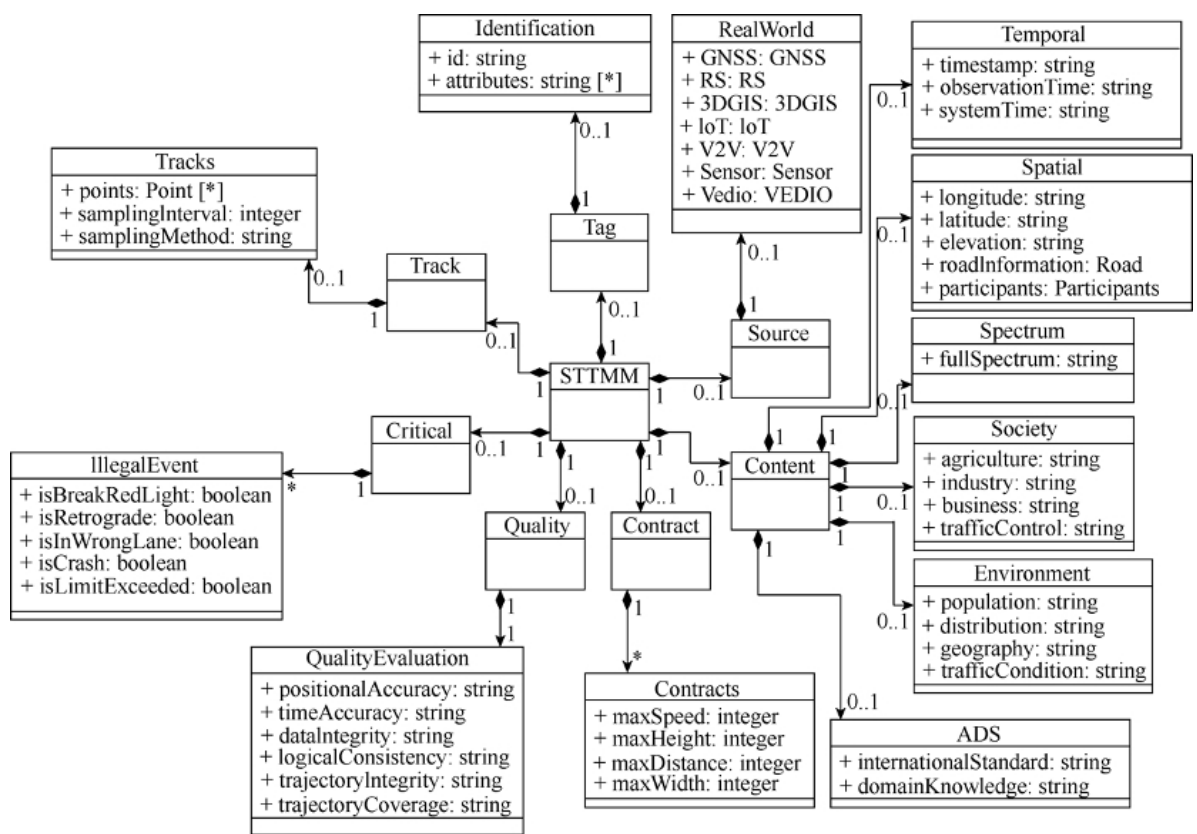

Figure 4 Meta-model of spatio-temporal trajectory data

The meta-model of spatio-temporal trajectory data provides support for the modeling of autonomous driving safety scenarios. With this meta-model, spatio-temporal trajectory data can be structured and stored to support the automatic instantiation of autonomous driving scenarios in a data-driven way.

\subsection{Management of high-quality spatio-temporal trajectory data}

High-quality spatio-temporal trajectory data is the core of autonomous driving safetycritical scenario modeling, which determines the accuracy and reliability of the data-driven model verification. Therefore, the collection, preprocessing, and quality evaluation model of spatio-temporal trajectory data are needed to ensure the data quality. Accurate collection and processing of spatio-temporal trajectory data is the primary task for providing high-quality data for the modeling of autonomous driving safety-critical scenarios. Secondly, because of the complicated source, inconsistent data formats, and missing data, the data fusion, data cleaning, and unified data representation should be carried out before the scenario modeling. Finally, a set of data quality evaluation methods are needed to ensure the high quality of the obtained spatio-temporal trajectory data. The specific implementation methods of Modules 1 and 2 shown in Figure 1 are as follows.

(1) Collection of spatio-temporal trajectory data

The accurate acquisition of high-quality data directly determines the authenticity and effectiveness of the autonomous driving safety-critical scenario. According to the data metamodeling method in Section 3.2, the information of the road network, where the autonomous vehicle is located is obtained by OpenStreetMap (OSM), and used to construct the road network 
of the autonomous driving scenario to perform the static environment modeling. GPS is used to obtain the real-time position and trajectory data of the autonomous vehicle ${ }^{[20]}$. During the GPS position, vehicle and pedestrian information around the autonomous vehicle is obtained by vehicle radar, cameras, sensors, and V2X that detect the objects in all directions around the vehicle, so as to form the spatio-temporal trajectory data. In this way, autonomous driving spatio-temporal trajectory data can be obtained.

(2) Preprocessing of spatio-temporal trajectory data

The collected spatio-temporal trajectory data should be preprocessed. Specifically, the similar and repeated data, the incomplete data, and the error data should be cleaned out ${ }^{[21]}$. There is a set of mature methods (e.g., ArcGIS) to process the map data downloaded from OSM. Due to the massive amount of characteristics, the trajectory data of autonomous vehicles obtained by GPS should be collected by a low-frequency sampling method ${ }^{[22]}$. However, this will cause certain errors between the GPS-positioned trajectory and the actual trajectory of the vehicle. Therefore, a map matching algorithm is used to correct the trajectory data and solve the problems of high redundancy, poor accuracy, and inconsistency ${ }^{[23]}$. Since video data needs to be used for analyzing the road structure and surrounding environment during the modeling of an autonomous driving vehicle, the video data is processed with the available mining methods (e.g., top-down method ${ }^{[24]}$ ). The sensors mainly obtain the data of surrounding environment, and there will be many redundant points and noise points. The sensor data now is mainly processed by Kalman filtering ${ }^{[25]}$.

(3) Quality evaluation model of spatio-temporal trajectory data

The quality of spatio-temporal trajectory data affects the accuracy of autonomous driving scenario modeling. Traditional quality evaluation methods of spatio-temporal data cannot effectively evaluate the data quality of autonomous driving, and thus it is necessary to establish a quality evaluation model for spatio-temporal trajectory data. On the basis of the existing quality evaluation methods of spatio-temporal data ${ }^{[22,26]}$, the spatio-temporal trajectory metadata structure of the autonomous vehicle, and the spatio-temporal trajectory data meta-model, this study abstracts six evaluation indicators to build a quality evaluation model for the spatiotemporal trajectory data of autonomous vehicles.

As shown in Figure 5, the quality evaluation model of spatio-temporal trajectory data includes 6 indicators: Time Accuracy (TA), Positional Accuracy $(P A)$, Data Accuracy $(D A)$, Temporal and Spatial Consistency (TSC), Trajectory Integrity (TI), and Sampling Frequency $(S F)$. These are the key attributes that reflect the domain knowledge, and the key indicators for evaluating the quality of spatio-temporal trajectory data in the autonomous driving field. Regarding the weighting of each indicator, this study combines the evaluation system and data evaluation methods of spatio-temporal data from other fields, and uses the expert scoring method and the analytic hierarchy process ${ }^{[27,28]}$ to obtain the weight of each indicator. At the same time, the fuzzy comprehensive evaluation method is used ${ }^{[29]}$ to solve the fuzzy uncertainty of the spatio-temporal trajectory data and make the evaluation result more reasonable. The evaluation formula corresponding to each indicator is given accordingly. Therefore, the data quality can be expressed as

$$
\begin{aligned}
Q= & W(T A) \times T A+W(P A) \times P A+W(D A) \times D A+W(T S C) \times T S C \\
& +W(T I) \times T I+W(S F) \times S F
\end{aligned}
$$

The evaluation model measures the quality of spatio-temporal trajectory data. With the quality evaluation model of spatio-temporal trajectory data, the data quality can be analyzed; the data with minor quality problems can be rectified, or the data with serious quality problems 
can be directly deleted. In the future work, we will optimize the evaluation model to make it more accurate and effective.

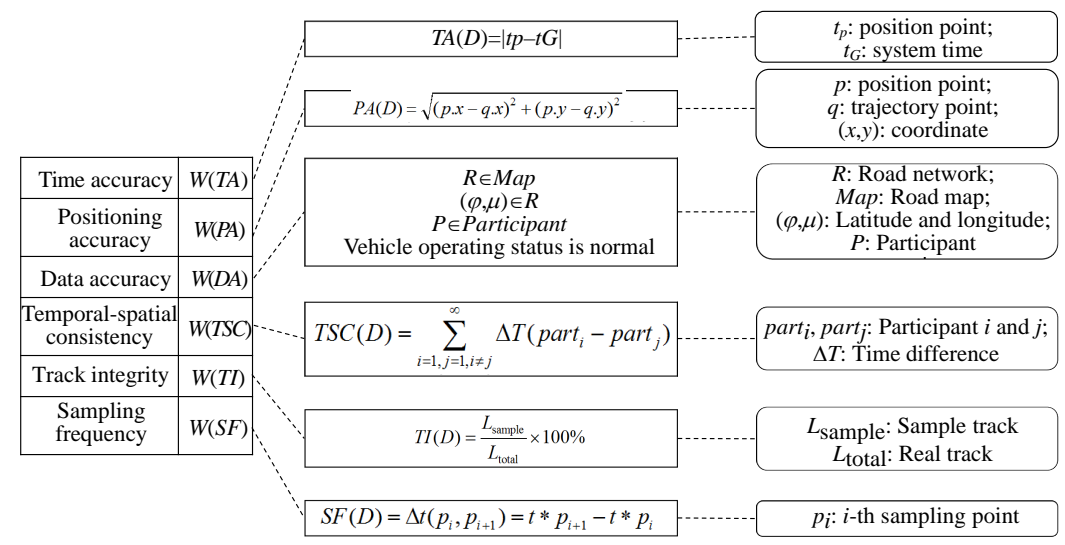

Figure 5 Data quality evaluation model of spatio-temporal trajectory data

\section{Autonomous Driving Safety Scenario Modeling Driven by STTD}

This section uses ADSML to discuss how to apply the meta-modeling method of spatiotemporal trajectory data to autonomous driving modeling. Through the modeling and analysis of spatio-temporal trajectory data and the automatic instantiation of the ADSML model with the high-quality data, we build an autonomous driving scenario model with spatio-temporal characteristics. ADSML is a modeling language oriented to the autonomous driving developed by our team, which not only provides the effective modeling technology for this field but also lays a foundation for building an autonomous driving system in a model-driven and data-driven collaborative development way.

\subsection{ADSML}

Scenario modeling is the basis for simulation, verification, and analysis of autonomous driving safety-critical scenarios. Modeling requires the design of a scenario modeling language for autonomous driving based on the data meta-model and the domain knowledge. Therefore, our team designed ADSML, which follows the principle of autonomous driving scenario metamodeling driven by spatio-temporal trajectory data. The modeler first analyzes the scenario that needs to be modeled, abstracts the elements that need to be modeled and their attributes from the spatio-temporal trajectory data meta-model, and uses ADSML to build the scenario model based on the abstraction results and support the model verification. One of the main features of ADSML is that it can realize the construction of scenario elements and the interactive modeling of Stochastic Hybrid Automata (SHA), which can support the synchronous interaction of visual modeling and formal modeling, and help designers construct the scenario models for formal verification. The modeling language has been implemented in open source tools based on the Meta Programming System (MPS).

The ADSML syntax is abbreviated as ADSML:=(Scenario, Contract, SHA), which contains three parts:

(1) Scenario:=(Map, Weather, RoadNetWork, Entity), which describes the static structural elements in the scenario. 
(2) Contract:=(Rule, Event). The contract module is associated with the rules of the scenario description module and part dynamic behavior of the entity. The dynamic behavior is described by event triggers, namely the state changes of certain elements in the scenario that is triggered at a certain condition.

(3) SHA describes the dynamic behavior of the entities in a scenario, which is associated with or dependent on the scenario description module and the contract module. It can well model the changes in state and the life cycle of the entities in the scenario, laying a foundation for the correctness analysis of the scenario behavior.

The specific syntax and semantics of ADSML can be found in another work of our team ${ }^{[14]}$. An example of ADSML modeling is shown in Figure 6.

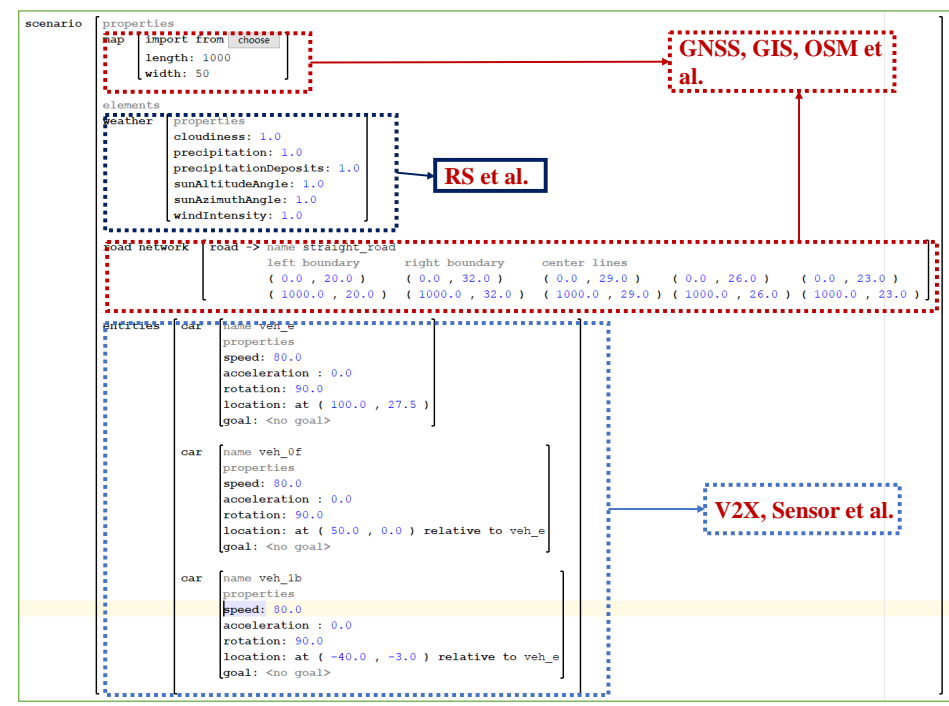

(a) Description example of overtaking scenario

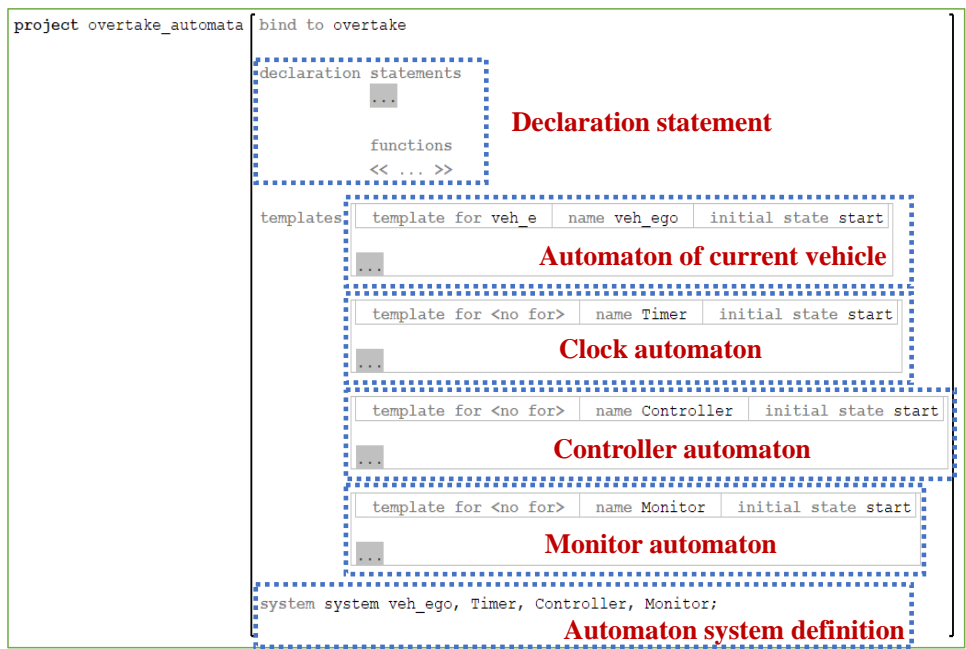

(b) SHA example of overtaking scenario 


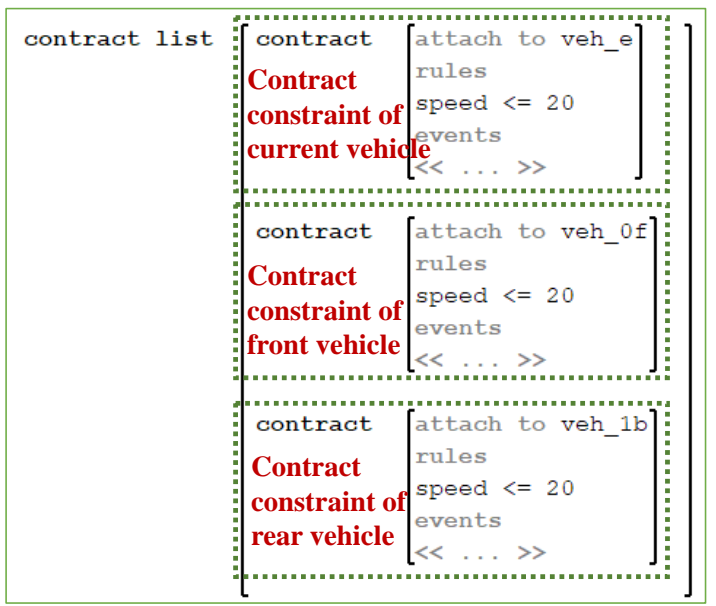

(c) Contract example of overtaking scenario

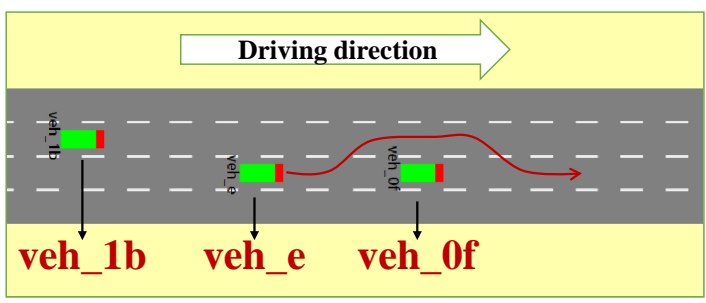

(d) Simulation model of overtaking scenario

Figure 6 Case study: Overtaking scenario modeling with spatio-temporal trajectory data-driven modeling approach

\subsection{Instantiation of autonomous driving scenario modeling based on ADSML}

This section uses ADSML to automatically instantiate the safety-critical scenario of automatic driving and construct an autonomous driving safety-critical scenario library.

The automatic instantiation of autonomous driving scenarios is performed in three steps.

Step 1. Collection of high-quality spatio-temporal trajectory data: First, according to the method given in Section 3.4, the high-quality real-time trajectory data of an autonomous vehicle is obtained after collection, preprocessing, and quality evaluation. Then, with the spatiotemporal trajectory data meta-model presented in Section 3.3, the spatio-temporal trajectory data is converted into structured data and stored as a data file in a readable XML format for subsequent scenario modeling and data analysis.

Step 2. Autonomous driving scenario modeling with ADSML: The spatio-temporal trajectory data is imported into ADSML according to the time-series relationship to generate the model. The data meta-modeling system described in Section 2.2 provides a spatio-temporal trajectory data flow for ADSML so to automatically instantiate the scenario model.

Step 3. Safety verification of scenario model: After the ADSML scenario instantiation, the method implemented by Module 7 shown in Figure 1 is used to abstract the SHA of autonomous driving from the vehicle journey to judge whether the autonomous vehicle is running in a certain predetermined mode. The occurrence of an illegal event mentioned in the meta-model of the 
spatio-temporal trajectory data in Section 3.3 may cause the system to enter a safety-critical state, prompting the system to report the abnormality to the supervisor.

The above three steps form a complete method for the automatic instantiation of autonomous driving safety scenarios modeled with high-confidence spatio-temporal trajectory data. The instantiated scenarios can be trained to predict when the autonomous vehicle will enter an unsafe state so as to ensure the safe operation of the autonomous driving system.

Based on the above instantiation method, ADSML is used to model the overtaking scenario of autonomous driving. The modeling performance is shown in Figure 6. Figure 6(a) shows a description example of ADSML scenario modeling, including map and road network (corresponding to the data source of GNSS, GIS, OSM), weather (corresponding to the data of $\mathrm{RS}$ ), entities (corresponding to information collected by V2X and autonomous vehicle). The entities include the current vehicle (veh_e), the front vehicle (veh_0f), and the rear vehicle (veh_1b). The vehicles veh_e and veh_0f are in the same lane while veh_1b is in another lane. In this scenario, veh_e needs to change to another lane to overtake veh_0f, and thus it is necessary to control the speed so that veh_e overtakes veh_0f without colliding with veh_1b. Figure 6(b) shows the SHA in the overtaking scenario, including the current autonomous vehicle (veh_ego), timer, controller, and monitor. Veh_ego defines the current operating state and attributes of the vehicle and simulates the state of the controlled vehicle veh_e, including forwarding, allowing/not allowing overtaking, acceleration/deceleration, returning to the original lane, and ending overtaking. Interaction with other models is performed through the channels and controllers to model the lane-changing and overtaking behavior. Figure 6(c) shows the contract module of the overtaking scenario (corresponding to the contract in the spatio-temporal trajectory data), and describes the rules and some dynamic properties that the three vehicles should obey in the scenario, with a speed limit of $20 \mathrm{~m} / \mathrm{s}$. The contract can also assist the modeling of the SHA. Figure 6(d) shows the visual simulation model of the overtaking scenario. The vehicle veh_e will overtake by switching lane along the red line. During the simulation process, the operating status of the autonomous vehicle will be judged in real time so as to provide extensive safety analysis and ensure a safe driving. Finally, through the result analysis of the scenario simulation model, whether and when the vehicle enters the unsafe state will be judged; UPPAAL is used for the verification analysis of the SHA. The above is an example of a case study of autonomous driving scenario with meta-modeling method.

With the automatic instantiation of the autonomous driving safety-critical scenario model, a large number of models can be generated for constructing an autonomous driving safety scenario library. This paper designs an automatic platform for generating an autonomous driving scenario library driven by spatio-temporal trajectory data. As shown in Module 6 in Figure 1, typical scenario models include straight road, T road, crossroad, and roundabout. For specific vehicle behavior scenarios, a corresponding safety scenario library can be built, such as acceleration, switching lane, overtaking, following, meeting, and accidents. The library of application scenarios as parks, airports, mining areas, parking lots, ports, highways, and urban roads can be built ${ }^{[30,31]}$. These scenarios play an important role in the subsequent actual vehicle testing and formal verification, and provide credible support for the development of autonomous driving technology.

\section{Related Work}

This paper studies the meta-modeling method for autonomous driving scenarios driven by spatio-temporal trajectory data. Related work mainly includes the application of spatiotemporal trajectory data, its meta-modeling, and the modeling method of autonomous driving safety scenarios. 
The application of spatio-temporal trajectory data mainly concentrates on the efficient storage and query, extraction, and update of road network information, and recognition of activity patterns ${ }^{[32,33]}$. There are few studies about the modeling verification based on spatiotemporal trajectory data. Zeng ${ }^{[26]}$ proposed a quality control and evaluation system of the spatial data, and analyzed the quality model, quality control method, and quality inspection and evaluation method of spatial data with consideration to the error sources. Yuan et al. ${ }^{[22]}$ integrated the driving data of all floating cars into floating car data, and proposed a quality evaluation model for floating car trajectory data, which measures the quality of data from 9 dimensions. The spatio-temporal trajectory data of autonomous vehicles is slightly different from the traditional big data ${ }^{[34]}$ whose work cannot be used for modeling and analysis in the field of autonomous driving. Therefore, we propose autonomous driving spatio-temporal trajectory data which includes not only the trajectory data of the autonomous vehicle but also the static information (spatial information, spectral information, humanistic information, social information) and the dynamic environmental information (e.g., traffic participant information) around the vehicle based on Zhang's previous work ${ }^{[2,3]}$. In this paper, the static environment information and dynamic behavior information of the autonomous vehicle during the driving process is integrated into spatio-temporal trajectory data with the modeling characteristics of the autonomous driving domain, which is used for the later modeling, simulation, and verification of the autonomous driving safety-critical scenario ${ }^{[35]}$.

The ideas of spatio-temporal metadata and meta-modeling have been widely used in safetycritical fields. Zhang et al. ${ }^{[3]}$ summarized the method of constructing spatio-temporal metadata into seven steps, formed and improved the high-confidence spatio-temporal metadata, and promoted the development of high-confidence software in the spatial information industry. Fatehah and Mezhuyev ${ }^{[36]}$ proposed a design meta-model and a process meta-model, and performed formally the modeling and verification of intelligent buildings by using the UPPAAL validator. The development and application of spatio-temporal metadata and meta-modeling technology has achieved preliminary success, whereas the available studies mostly focus on the application in intelligent buildings and geographic information ${ }^{[37]}$. There is less related work on metadata and meta-modeling in the field of autonomous driving. Therefore, based on the domain knowledge of autonomous driving, this paper constructs the metadata structure of the spatio-temporal trajectory, the metamodel of spatio-temporal trajectory data, and the quality evaluation method tailored to the characteristics of the autonomous driving domain, which lays a foundation for the model-driven and data-driven collaborative development of intelligent systems. In addition, it can also be used to guide the construction of data-driven scenario models in this field, which is the difference between this paper and the existing ones.

The existing modeling and simulation testing techniques for the autonomous driving safety scenario have their own advantages and different concerns. Association for Standardization of Automation and Measuring systems (ASAM) ${ }^{[16]}$ organized two projects: OpenScenario ${ }^{[17]}$ and OpenDrive ${ }^{[18]}$. OpenScenario is an open file format describing the dynamic content in driving simulation applications, and OpenDrive supports the static content. In addition, Seshia's research group at UC Berkeley designed a driving scenario description language Scenic ${ }^{[10]}$ that can generate simulation scenarios on a GTAV simulator with the help of DeepGTAV ${ }^{[38]}$. Rodrigo et al.$^{[9]}$ proposed the open source domain-specific language GeoScenario for the representation of autonomous driving scenarios, which is simple and extensible and mainly used for constructing the infrastructure of a driving simulator. CommonRoad proposed by Althoff et al ${ }^{[39]}$ created a specific file format for the description of autonomous driving scenarios, while there was no domain-specific description language for scenario description. Bach et al. ${ }^{[40]}$ used the graphical modeling technology for scenario modeling and proposed a model-based development and test 
method for autonomous driving functions, whereas the scenario elements that can be modeled are limited. In short, despite the certain advantages, the existing modeling of autonomous driving has some deficiencies, such as a lack of a readable modeling description language and a difficulty in using formal methods to verify and analyze the safety of the driving scenarios. This paper puts forward a autonomous driving scenario modeling method driven by spatio-temporal trajectory data, constructs the metadata model in the ADS field, and introduces the high-quality spatio-temporal trajectory data to autonomous driving scenario modeling. With the ADSML language used for the scenario modeling, we can reconstruct the autonomous driving scenario accurately and realize the real-time evaluation of the safety of driving scenarios by using the formal methods.

\section{Conclusions and Prospects}

Technologies such as autonomous driving, V2X, artificial intelligence, and big data have achieved unprecedented development. However, the explosive growth of data and the openness and complexity of the operating environment have exposed the safety problems of such systems. In addition, the rapid development of technologies such as RS, GIS, GNSS, and sensors has made it convenient for people to obtain the spatio-temporal trajectory data. However, how to realize the safety scenario modeling of the autonomous driving system based on such massive data is also one of the major challenges. In response to the above problems, this paper proposes to unify and integrate the spatio-temporal trajectory data and develops a spatio-temporal trajectory data meta-modeling method oriented to autonomous driving, which lays a foundation for the modeling of safety scenarios and facilitates the realization of data unification, processing, and reuse. Meanwhile, this paper incorporates our previous work-ADSML, and discusses in detail how to use ADSML to realize the scenario instantiation. The case study shows that this method can generate the abstraction models of safety scenarios. The above work provides a spatiotemporal trajectory data-driven safety scenario modeling method for abstract modeling of safety scenarios, which can effectively model autonomous driving scenarios and construct a scenario model library, providing a convenient method for the simulation and verification of autonomous driving.

In the next stage of work, we will focus on how to use the STL language to describe the behavior constraints in the scenario and combine it with the statistical model detection technology to analyze and verify the random behavior in the autonomous driving scenario. Particularly, we will try to explore how to combine the runtime verification with statistical model detection to analyze the correctness of the STL specification, which is of great significance to verify the correctness of autonomous driving scenario model.

\section{References}

[1] Yao D, Zhang C, Huang JH, et al. Semantic understanding of spatio-temporal data: Technology \& application. Ruan Jian Xue Bao/Journal of Software, 2018, 29(7): 2018-2045. http://www.jos.org.cn/ 1000-9825/5576.htm [doi: 10.13328/j.cnki.jos.005576].

[2] Zhang L, Zhang CX, Du DH, et al. Constraint expression method of spatial information and spatial big data. Shanghai, CN110532341B, 2020-11-03 (in Chinese).

[3] Zhang L, Zhang CX, Du DH, et al. Spatial information spatial metadata construction metho. Shanghai, CN110532340A, 2019-12-03 (in Chinese).

[4] Tesla accident (in Chinese). https://36kr.com/p/871536866489736.

[5] Nidhi K, Paddock SM. Driving to safety: How many miles of driving would it take to demonstrate autonomous vehicle reliability. Technical Report, Santa Monica: RAND Corporation, 2016. https: //www.rand.org/pubs/research_reports/RR1478.html 
[6] Zhu B, Zhang PX, Zhao J, et alv. Review of scenario-based virtual validation methods for automated vehicles. China Journal of Highway and Transport, 2019, 32(6): 1-19.

[7] de Gelder E, Paardekooper JP. Assessment of automated driving systems using real-life scenarios. Proc. of the 2017 IEEE Intelligent Vehicles Symp. (IV). IEEE, 2017. 589-594.

[8] Zhao D, Guo Y, Jia YJ. Trafficnet: An open naturalistic driving scenario library. Proc. of the 20th IEEE Int'1 Conf. on Intelligent Transportation Systems (ITSC). IEEE, 2017. 1-8.

[9] Queiroz R, Berger T, Czarnecki K. GeoScenario: An open DSL for autonomous driving scenario representation. Proc. of the 2019 IEEE Intelligent Vehicles Symp. (IV). IEEE, 2019. 287-294.

[10] Fremont DJ, Dreossi T, Ghosh S, et al. Scenic: A language for scenario specification and scene generation. Proc. of the 40th ACM SIGPLAN Conf. on Programming Language Design and Implementation. 2019. 63-78.

[11] OpenStreetMap. https://www.openstreetmap.org/.

[12] Meta-Object Facility (MOF). https://en.wikipedia.org/wiki/Meta-Object_Facility.

[13] UML OMG. OMG (2017) Unified Modeling Language ${ }^{\circledR}$ (OMG UML $\left.{ }^{\circledR}\right)$ Version 2.5. 2017. https: //www.omg.org/spec.

[14] Wang Y. Modeling, simulation and verification of autonomous driving scenario based on model-driven [MS. Thesis]. Shanghai: East China Normal University, 2020.

[15] Menzel T, Bagschik G, Maurer M. Scenarios for development, test and validation of automated vehicles. Proc. of the 2018 IEEE Intelligent Vehicles Symp. (IV). IEEE, 2018. 1821-1827.

[16] Association for Standardisation of Automation and Measuring Systems. https://www.asam.net/.

[17] OpenScenario. https://www.asam.net/standards/detail/openscenario.

[18] OpenDRIVE. https://www.opendrive.com.

[19] Bartocci E, Deshmukh J, Donzé A, et al. Specification-based monitoring of cyber-physical systems: A survey on theory, tools and applications. In: Lectures on Runtime Verification. Cham: Springer-Verlag, 2018. 135-175.

[20] Urmson C, Anhalt J, Bagnell D, et al. Autonomous driving in urban environments: Boss and the urban challenge. Journal of Field Robotics, 2008, 25(8): 425-466.

[21] Gao Q, Zhang FL, Wang RJ, et al. Trajectory big data: A review of key technologies in data processing. Ruan Jian Xue Bao/Journal of Software, 2017, 28(4): 959-992. http://www.jos.org.cn/1000-9825/ 5143.htm [doi: 10.13328/j.cnki.jos.005143].

[22] Yuan H. Research on modeling spatiotemporal trajectories and spatiotemporal proximity analysis for time geographic analysis [Ph.D. Thesis]. Wuhan: Wuhan University, 2018.

[23] Shen L, Stopher PR. Review of GPS travel survey and GPS data-processing methods. Transport Reviews, 2014, 34(3): 316-334.

[24] Buch N, Velastin SA, Orwell J. A review of computer vision techniques for the analysis of urban traffic. IEEE Trans. on Intelligent Transportation Systems, 2011, 12(3): 920-939.

[25] Yu C, Zhang LJ, Jin H. Research progress and trend of big data-driven intelligent transportation system. Wu Lian Wang Xue Bao/Chinese Journal on Internet of Things, 2018, 2(1): 56-63. [doi: 10.11959/j.issn.2096-3750.2018.00041]

[26] Zeng YW. Research on spatial data quality control and evaluation technique system [Ph.D. Thesis]. Wuhan: Wuhan University, 2004. [doi: 10.7666/d.Y698781]

[27] Zhang J, Zhou Y. New software trustworthiness attribute weight distribution method based on attribute affection and importance. Ji Suan Ji Ying Yong Yan Jiu/Application Research of Computers, 2016, 33(5): 1390-1394.

[28] Tao HW. Research on the measurement models of software trustworthiness based on attributes [Ph.D. Thesis]. Shanghai: East China Normal University, 2011. [doi: 10.7666/d.y1905206]

[29] Hu SW. GIS quality evaluation and reliability analysis based fuzzy theory [Ph.D. Thesis]. Wuhan: Wuhan University, 2004.

[30] Zhang N, Yang YR, You TY, et al. Development trends in China high-level autonomous driving from 
2020 to 2023. Proc. of the EO Intelligence. 2020. 1-54.

[31] Holographic smart scene library derived from real traffic data. http://www.scenarios.cn/html/index. html.

[32] Yang W. Structured processing and behavioral semantic perception of spatiotemporal trajectory data [Ph.D. Thesis]. Wuhan: Wuhan University, 2019.

[33] Yang TT. The real-time traffic status acquirement and system assessment based on Taxi GPS trajectory data [MS. Thesis]. Shanghai: East China Normal University, 2016.

[34] Xu JJ, Zheng K, Chi MM, et al. Trajectory big data: Data, applications and techniques. Tong Xin Xue Bao/Journal on Communications, 2015, 36(12): 97-105. [doi: 10.11959/j.issn.1000-436x.2015318]

[35] Xin B. Analysis approach of assisted driving safety scene based on learning and model checking [MS. Thesis]. Shanghai: East China Normal University, 2020.

[36] Fatehah M, Mezhuyev V. Design and process metamodels for modelling and verification of safetyrelated software applications in smart building systems. Proc. of the 6th Int'l Conf. on Information Technology: IoT and Smart City (ICIT 2018). New York: Association for Computing Machinery, 2018. 60-64. [doi: 10.1145/3301551.3301577]

[37] Chen JN, Li J. A method of sharing heterogeneous data in smart cities based on meta-models. CN104462244A, 2015-03-25 (in Chinese).

[38] DeepGTAV. https://github.com/aitorzip/DeepGTAV.

[39] Althoff M, Koschi M, Manzinger S. Commonroad: Composable benchmarks for motion planning on roads. Proc. of the 2017 IEEE Intelligent Vehicles Symp. (IV). 2017. 719-726.

[40] Bach J, Otten S, Sax E. Model based scenario specification for development and test of automated driving functions. Proc. of the 2016 IEEE Intelligent Vehicles Symp. (IV). IEEE, 2016. 1149-1155.

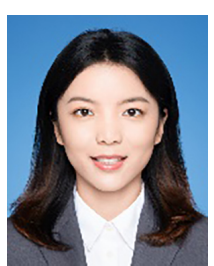

Menghan Zhang master, $\mathrm{CCF}$ student member. Her research interest is the security-critical scenario modeling.

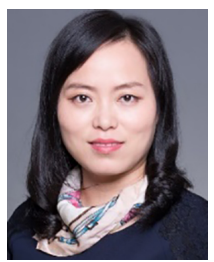

Dehui Du Ph.D., Professor, Ph.D. supervisor, CCF professional member. Her research interests include the trusted software, modeling and verification of information physical fusion system, and security and reliability theory and verification of artificial intelligence.

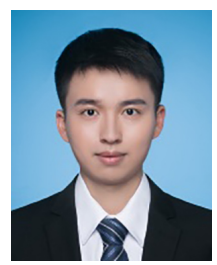

Yao Wang master, CCF student member. His research interest is the trusted software.

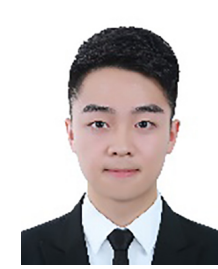

Mingzhuo Zhang master, CCF student member. His research interest is the trusted software.

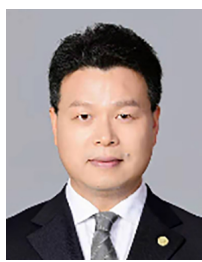

Lei Zhang Ph.D., Professor, $\mathrm{Ph} . \mathrm{D}$. supervisor, CCF senior member. His research interests include the physical information fusion and data intelligence applications.

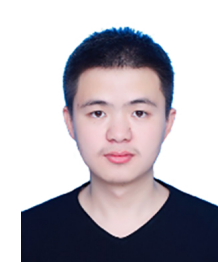

Wentao Zhou maste. His research interest is the trusted software. 